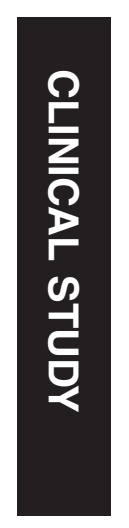

\title{
Silicone oil versus gas tamponade for giant retinal tear-associated fovea-sparing retinal detachment: a comparison of outcome
}

${ }^{1}$ Vitreoretinal Unit, Moorfields Eye Hospital, London, UK

${ }^{2} \mathrm{NIHR}$ Clinical Research Facility at Moorfields Eye Hospital, London, UK

${ }^{3}$ Frimley Health NHS Foundation Trust, Camberley, UK

${ }^{4}$ Ophthalmology Department, Southend University Hospital, Southend on Sea, UK

${ }^{5}$ First Ophthalmology Department, University of Athens, Athens, Greece

Correspondence: PJ Banerjee, Clinical Research Fellow, Vitreoretinal Service, Moorfields Eye Hospital, 162 City Road, London EC1V 2PD, UK Tel: +44 (0)2075662251; Fax: $+44(0) 2076086925$ E-mail: philipbanerjee@ doctors.org.uk

Received: 31 December 2016

Accepted in revised form: 30 June 2017

Published online:

11 August 2017

Presented at Annual Association for Research in Vision and Ophthalmology Meeting, Fort Lauderdale, Florida, USA, May 2012.

\section{Abstract}

Purpose Silicone oil is the most common choice of tamponade agent used when managing a giant retinal tear (GRT). Concern exists regarding its potential detrimental effect on vision. We herein report on visual and surgical outcomes of all patients treated at Moorfields Eye Hospital for a GRT over a five year period. We further analysed a subgroup of fovea-sparing retinal detachments (RD) treated by pars plana vitrectomy (PPV) with either silicone oil or gas tamponade.

Methods Retrospective comparative, nonrandomised, cross-sectional review of patients with a new diagnosis of GRT from 1 September 2005 to 31 August 2010 Results One hundred twenty four eyes of 118 patients were identified with mean age of 45.5 years and median follow-up of 24 months. Subgroup analysis of foveasparing RDs (Silicone Oil $n=49$, Gas $n=15$ ) revealed visual loss $(\geq 2$ Snellen Lines of vision) in $49.0 \%(n=24)$ of patients managed with oil compared to $13.3 \%(n=2)$ of gas patients $(P=0.019)$. In all, $73.3 \%(n=11)$ in the gas group achieved a final vision of $6 / 12$ or better, compared to $36.7 \%(n=18)$ in the oil group $(P=0.031)$. No difference was observed in eventual or primary anatomical success rate (100 vs $93.9 \%$, and 66.7 vs $79.6 \%$, gas vs oil, respectively, $(P>0.1))$. Postoperative complications were absent in $66.7 \%(n=10)$ of gas patients compared with $14.3 \%(n=7)$ of oil patients $(P=0.002)$. Multiple variable linear regression determined tamponade choice as the only variable predictive of final visual acuity $(P=0.046)$.

Conclusion Eyes with fovea-sparing GRT-related RDs managed with gas achieved a better visual outcome with fewer
PJ Banerjee ${ }^{1,2,3}$, A Chandra ${ }^{1,2,4}$, P Petrou ${ }^{1,5}$ and DG Charteris ${ }^{1,2}$ postoperative complications and no significant difference in anatomical success. A multicentre approach to investigate this further is advised.

Eye (2017) 31, 1302-1307; doi:10.1038/eye.2017.167; published online 11 August 2017

\section{Introduction}

A giant retinal tear (GRT) is defined as a full thickness retinal break of three or more clock hours in circumference in the presence of a detached posterior hyaloid. ${ }^{1-3}$ The incidence of proliferative vitreoretinopathy (PVR) in GRTrelated retinal detachments (RDs) is greater than in non-GRT-related RDs, with reported incidences ranging from 11 to $41 \%$, and comparatively poorer associated surgical outcomes. ${ }^{2,4-11}$ Globally, silicone oil remains the most widely used tamponade agent when managing GRTs, $5,12-19$ employed in up to $85 \%$ of cases in the UK. ${ }^{4}$ In addition to its well documented side effects, which include glaucoma and keratopathy, concern exists regarding its potential detrimental effect on vision particularly after its removal. ${ }^{20-25}$ The mechanism of visual loss on silicone oil removal remains unexplained and is of particular concern in patients whose fovea remains attached.

A small randomized controlled clinical trial comparing the visual and surgical outcomes in patients with a GRT and Grade C proliferative vitreoretinopathy, found no difference between patients treated with silicone oil or perfluoropropane $\left(\mathrm{C}_{3} \mathrm{~F}_{8}\right)$ at 5 years. ${ }^{26}$

This study was undertaken to (a): report on the visual and surgical outcomes of all patients treated at Moorfields Eye Hospital for a GRT over a 5-year period and (b) to compare the 
outcomes of a subgroup of patients with fovea-sparing retinal detachments treated by pars plana vitrectomy and either silicone oil or gas tamponade; this analysis being undertaken to exclude the potential confounding effect of foveal detachment.

\section{Materials and methods}

This study was a retrospective, comparative, nonrandomised cross-sectional review. Following local research and management committee approval, a retrospective electronic and paper case note review was performed on all patients who presented to Moorfields Eye Hospital between 1 September 2005 and 31 August 2010 with a new diagnosis of GRT.

Cases were identified using specific electronic search codes or by the documentation of a GRT in electronic patient records consisting of: outpatient clinic records, primary care correspondences and operative records.

Baseline demographic and ocular characteristics, operative management, and visual and surgical outcomes were recorded.

\section{Statistical methods}

Fisher's exact test was used to compare categorised visual and surgical outcome in eyes with fovea-sparing retinal detachments using IBM SPSS Statistics Version 24.0 (IBM, UK Ltd). Visual outcome measures included (a) the proportion of eyes achieving a final vision of $\geq 6 / 12$ and (b) the proportion of patients losing two or more lines on the Snellen visual acuity chart when compared to baseline vision. Surgical outcome measures were (a) the proportion of patients achieving eventual anatomical success with the absence of internal tamponade, (b) the proportion of patients achieving primary success with the absence of internal tamponade and (c) the proportion of patients suffering postoperative complications.

Forward stepwise multiple variable linear regression analysis was performed to determine independent baseline variables associated with visual outcome in eyes with fovea-sparing retinal detachments.

\section{Results}

This review included 124 eyes of 118 patients who presented to Moorfields Eye Hospital over the study period with a median follow-up of 24 months (range 2-72 months). Review of notes occurred at least 12 months post GRT repair. Paper or electronic case notes retrieval was achieved for all cases.

\section{Baseline characteristics}

Baseline characteristics are summarised in Table 1. The majority of the cohort were male, with associated trauma reported in approximately one fifth of patients. High myopia was present in approximately one quarter of patients in whom the refractive status was known.

Median presenting vision was 6/24 (range, 6/5-light perception). Approximately $40 \%$ of eyes presented with a visual acuity of $\geq 6 / 12$ with one third seeing worse than $6 / 60$ at presentation. One quarter of patients were pseudophakic.

Retinal detachment (RD) was present in $92 \%$ of eyes at presentation, over half of which $(73,58.9 \%)$ were foveasparing. Large GRTs ( $>180^{\circ}$ degrees) were present in one quarter of eyes, and established PVR (Grade C) ${ }^{27}$ was documented in $10 \%$ of eyes at presentation.

Visual acuity in the fellow eye was $>6 / 12$ in the majority of patients $(89.5 \%)$.

\section{Management}

Data regarding the primary management of affected and fellow eyes are summarised in Table 2. Pars plana vitrectomy was the preferred choice of primary

Table 1 Baseline characteristics at presentation

\begin{tabular}{lc}
\hline Characteristics at presentation & $\mathrm{N}(\%)$ \\
\hline Eyes & 124 \\
Patients & 118 \\
Male & $106(89.8)$ \\
Mean age (years) & 45.5 \\
Predisposing conditions & \\
Trauma & $27(22)$ \\
Myopia $>$-6DS & 33 of $91(27)$ \\
Hereditary Vitreoretinopathies & $9(7.5)$ \\
Presenting VA $\geq 6 / 12$ & $54(43.5)$ \\
Presenting VA<6/60 & $40(32.3)$ \\
Lens status & \\
Phakic (Clear) & $71(57.3)$ \\
Cataract & $17(13.7)$ \\
Pseudophakic & $30(24.2)$ \\
Aphakic & $5(4.0)$ \\
Dislocated IOL & $1(0.8)$ \\
Retinal detachment & \\
Fovea attached & \\
GRT $\geq 180^{\circ}$ & $73(58.9)$ \\
PVR-Grade C & $31(25.0)$ \\
Fellow eye & $12(10.0)$ \\
VA $\geq 6 / 12$ & \\
VA <6/60 & \\
Previous/current GRT & $111(89.5)$ \\
Previous/current non-GRT RD & $6(4.8)$ \\
\hline & $5(4.0)$ \\
& $1(0.8)$ \\
\hline
\end{tabular}

Data expressed as $n$ (\%) of 124 eyes of 118 patients. 
Table 2 Data on primary management of affected and fellow eyes

\begin{tabular}{lc}
\hline Management & $\mathrm{N}(\%)$ \\
\hline Affected eyes (procedure) & \\
None & $2(1.6)$ \\
Vitrectomy & $112(90.3)$ \\
Laser/cryopexy alone & $9(7.3)$ \\
CryoBuckle & $1(0.8)$ \\
Localised retinopexy & 51 of $112(45.5)$ \\
$360^{\circ}$ Laser retinopexy & 60 of $112(27)$ \\
Cryopexy & $86(76.8)$ \\
& \\
Tamponade & \\
Silicone oil & 94 of $112(83.9)$ \\
Perfluoropropane $\left(\mathrm{C}_{3} \mathrm{~F}_{8}\right)$ & 17 of $112(15.2)$ \\
Sulphurhexaflouride $\left(\mathrm{SF}_{6}\right)$ & 1 of $112(0.9)$ \\
Fellow eyes (procedures) & \\
None & 103 of $110(93.6)$ \\
$360^{\circ}$ Treatment & \\
$360^{\circ}$ Laser & 3 of $110(2.7)$ \\
$360^{\circ}$ Cryotherapy & 0 \\
Local retinopexy/cryopexy & 4 of $110(3.6)$ \\
\hline
\end{tabular}

Data expressed as $n(\%)$ of 124 eyes of 118 patients unless otherwise stated

management in over $90 \%$ of cases. In all, $27 \%$ of affected eyes were treated with $360^{\circ}$ barrier laser retinopexy, intraoperatively. A small number of cases $(n=9)$ were treated non surgically as there was no concomitant neurosensory detachment. One patient, initially diagnosed as a retinal dialysis, was managed primarily with a scleral buckle and cryopexy with subsequent augmented barrier laser retinopexy. The retina remained attached at 31 months with a documented visual acuity of $6 / 9$.

A total of 110 fellow eyes were eligible for prophylactic treatment at the time of their affected eye GRT. Most fellow eyes were not treated prophylactically (93.6\%), with only three eyes treated with $360^{\circ}$ prophylaxis and two eyes treated for local pathology. None of these eyes subsequently developed an RD over a median follow-up period of 24 months (range 5-42 months). Nine of 110 fellow eyes detached over the study follow-up period; all occurring within 22 months of their first eye GRT.

\section{Outcome}

Outcome data were available for 115 out of the 122 treated eyes and is summarized in Table 3. Patients with no outcome data or follow-up of $<2$ months were excluded. The median follow-up was 24 months with a range from 2 to 72 months. Eventual anatomical success rate was defined as an attached retina in the absence of an internal tamponade agent, and was achieved in $91.3 \%$ of eyes. In all, $79.1 \%$ of cases were successful at the first
Table 3 Postoperative outcome of 115 eyes

\begin{tabular}{lc}
\hline Postoperative outcome & $\mathrm{N}(\%)$ \\
\hline Median follow-up (months) & 24 \\
Visual acuity & $50(43.5)$ \\
$\geq 6 / 12$ & $23(20.0)$ \\
$<6 / 60$ & \\
& \\
Anatomic & $105(91.3)$ \\
Eventual success & $91(79.1)$ \\
Primary success & $4(3.5)$ \\
Silicone Oil in situ & \\
Postoperative complications & \\
Cataract (of phakic eyes) & $30(26)$ \\
Prolonged raised IOP/Glaucoma & $23(20)$ \\
Epiretinal membrane & $16(14)$ \\
Uveitis & 9 of $110(8.2)$ \\
Fellow Eye RD & \\
\hline Data expressed as $n$ (\%) of 115 eyes unless otherwise stated &
\end{tabular}

procedure. The proportion of patients achieving the Driving Vehicle License Association (DVLA) visual standards for driving $(\geq 6 / 12)$ was $43.5 \%$, with $20 \%$ of eyes seeing worse than $6 / 60$ at the time of their final follow-up.

The commonest postoperative complication was cataract, occurring in two thirds of phakic eyes (65.9\%). Prolonged elevated intraocular pressure (IOP) or glaucoma was present in one quarter $(26.1 \%)$ of eyes. Six eyes required filtration or aqueous shunt surgery over the period studied.

\section{Subgroup analysis of patients with fovea-sparing retinal detachments managed with silicone oil or gas tamponade}

Sixty eight eyes with fovea-sparing retinal detachments and managed by vitrectomy with either gas or silicone oil tamponade were analysed as a subgroup. Surgical and visual outcomes were compared. Table 4 summarizes the baseline characteristics of both groups. Expansile gas was the choice of primary tamponade agent in 16 (23.5\%) eyes, and the remaining 52 eyes $(77.5 \%)$ were managed with silicone oil. Perfluoropropane was the expansile gas of choice in 15 out of the 16 eyes, with sulpurhexaflouride used in one patient. Baseline demographics were similar in terms of age and sex. A median visual acuity of 6/9 was recorded in both groups. There was a higher incidence of high myopia (27.5\%), trauma (19.2\%), larger $\left(>180^{\circ}\right)$ GRTs $(26.9 \%)$ and PVR Grades A or B $(11.5 \%)$ in eyes managed with silicone oil compared to the gas group where rates were $15.4,12.5,6$ and $6 \%$, respectively. There were no documented cases of established PVR (Grade C) at baseline in either group. In all, $90.2 \%$ of GRTs managed with oil extended inferiorly (ie below 8 or 4 o'clock $^{28}$ ) 
Table 4 Baseline Characteristics of eyes with fovea-sparing RDs managed by silicone oil or gas tamponade

\begin{tabular}{lccc}
\hline $\begin{array}{l}\text { Baseline characteristics of fovea- } \\
\text { sparing retinal detachments }\end{array}$ & $\begin{array}{c}\text { Gas } \\
\text { tamponade }\end{array}$ & $\begin{array}{c}\text { Oil } \\
\text { tamponade }\end{array}$ & $\begin{array}{c}\mathrm{P} \\
\text { Value }\end{array}$ \\
\hline Patients & 16 & 52 & \\
Mean age (years) & 46.4 & 45.4 & \\
Males & $13(81.3)$ & $49(94.2)$ & \\
& & & \\
Predisposing conditions & $2(12.5)$ & $10(19.2)$ & 0.71 \\
$\quad$ Trauma & 2 of 13 & 11 of 40 & 0.48 \\
Myopia $\geq-6$ & $(15.4)$ & $(27.5)$ & \\
& 0 & $5(9.6)$ & \\
Hereditary vitreoretinopathies & & & \\
& & & \\
Clinical characteristics & $6 / 9$ & $6 / 9$ & \\
Median presenting VA & & & \\
& & & \\
Lens Status & $5(31.3)$ & $14(26.9)$ & \\
Pseudophakic & & & \\
& $1(6)$ & $14(26.9)$ & 0.1 \\
Retinal detachment & $1(6)$ & $6(11.5)$ & 0.68 \\
GRT > 180 & $5(33.3)$ & $46(90.2)$ & $<\mathbf{0 . 0 1}^{\text {a }}$ \\
PVR Grade A or B & & & \\
Inferior Tear (GRT extends & &
\end{tabular}

Data expressed as $n(\%)$ of 16 eyes in gas group and 52 eyes in oil group unless otherwise specified. abold type indicates statistical significance.

compared to only one third (33.3\%) of those managed with expansile gas $(P<0.01)$.

\section{Outcome analysis}

Visual and surgical outcomes were available in 15 of the 16 patients in the gas group, and 49 of 52 patients treated with oil. Comparisons between the groups are summarized in Table 5. Vision was categorized into the proportion of patients achieving $\geq 6 / 12$ (driving vision), and those patients suffering visual loss (defined as a drop of $\geq 2$ Snellen lines when comparing final visual acuity to that at presentation). In all, $73 \%$ of patients in the gas group achieved driving vision compared to only $37 \%$ in the oil group ( $P=0.0194$, Fisher's exact). Fewer patients lost vision in the gas group compared to the oil group, $13 \%$ and $48 \%$, respectively $(P=0.031)$. Regarding surgical outcomes, there was no statistical difference in the proportion of patients achieving eventual anatomical success, with 100 and $93.9 \%$ in the gas and oil group, respectively $(P>0.1)$. Primary success was achieved in $66.7 \%$ of patients treated with gas, compared to $79.6 \%$ in which oil was used $(P>0.1)$.

Patients in whom silicone oil was used as a primary tamponade agent underwent routine removal at 35 months post primary procedure. Seven eyes required further surgery following redetachment after primary oil removal; six of the seven required repeat surgery with oil,
Table 5 Outcome comparison of eyes with fovea-sparing RDs managed with either gas or oil

\begin{tabular}{|c|c|c|c|}
\hline Outcome Analysis & $\begin{array}{c}\text { Gas tamponade } \\
\quad(\mathrm{n}=15)\end{array}$ & $\begin{array}{l}\text { Oil tamponade } \\
\quad(\mathrm{n}=49)\end{array}$ & $\mathrm{P}$ value \\
\hline \multicolumn{4}{|l|}{ Vision } \\
\hline$\geq 6 / 12^{*}$ & $11(73.3)$ & $18(36.7)$ & 0.031 \\
\hline Vision Loss* & $2(13.3)$ & $24(49.0)$ & 0.019 \\
\hline \multicolumn{4}{|l|}{ (Loss of $\geq 2$ snellen lines) } \\
\hline \multicolumn{4}{|l|}{ Surgical } \\
\hline $\begin{array}{l}\text { Eventual anatomical } \\
\text { success }\end{array}$ & $15(100)$ & $46(93.9)$ & $>0.1$ \\
\hline \multicolumn{4}{|c|}{ Number of ops to achieve success: } \\
\hline 1 & $10(66.7)$ & 39 (79.6) & $>0.1$ \\
\hline 2 & $3(20)$ & $5(10.2)$ & \\
\hline 3 & $2(13.3)$ & $1(2.0)$ & \\
\hline 4 & 0 & $1(2.0)$ & \\
\hline \multicolumn{4}{|l|}{ Complications } \\
\hline $\mathrm{Nil}^{*}$ & $10(66.7)$ & $7(14.3)$ & 0.002 \\
\hline $\begin{array}{l}\text { Cataract (of phakic } \\
\text { eyes) }\end{array}$ & 4 of $10(40)$ & 18 of 35 (51.4) & $>0.1$ \\
\hline Uveitis & $2(13.3)$ & $8(16.3)$ & $>0.1$ \\
\hline $\begin{array}{l}\text { Prolonged elevated } \\
\text { IOP/glaucoma* }\end{array}$ & $1(6.7)$ & $16(32.7)$ & 0.047 \\
\hline
\end{tabular}

Data expressed as $n(\%)$ of 15 eyes in gas group and 49 eyes in oil group unless otherwise stated. Bold type indicates statistical significance. *Indicates statistical significance.

where one eye re-attached with repeat surgery and perfluoropropane gas. In the gas group, considering the 5 eyes where primary success was not achieved, four required repeat surgery with silicone oil, and one achieved subsequent successful surgery with sulfahexafluoride.

Postoperative complications were absent in $66.7 \%$ of gas-treated eyes compared with only $14 \%$ in eyes treated with oil $(P=0.002)$.

The aforementioned comparisons may be influenced by a disproportionate weighting of baseline variables associated with poorer outcomes in eyes treated with silicone oil, for example, PVR, larger GRTs $\left(>180^{\circ}\right)$, hereditary vitreoretinopathies, inferior tear position. Therefore, adopting final visual acuity (converted from Snellen to LogMAR) as the dependent variable, a forward stepwise multiple variable linear regression analysis was performed including all baseline variables in Table 4 . The final model fitted contained tamponade choice as the only independent variable significantly associated with visual outcome $(P=0.046)$.

Unexplained visual loss (defined as a drop of two or more Snellen lines) following oil removal without recovery was observed in 10 patients $(20.4 \%)$ and was the most common cause of poor visual outcome in the oil group. Advanced glaucomatous optic neuropathy $(n=9)$ was the next most common cause for poor vision in eyes managed with silicone oil. 


\section{Discussion}

Baseline characteristics and management data are comparable to recently published reports from a nationwide prospective epidemiological study. ${ }^{4}$ Visual and surgical outcomes of patients attending our unit over the 5-year period studied are also similar. ${ }^{4}$

Considering the prophylactic treatment of fellow eyes, there is a clear difference in practice at our institution compared to the rest of the UK. Prophylactic treatment of $360^{\circ}$ was performed in only 3 out of $110(2.7 \%)$ eligible cases (2 patients were treated for local pathology) compared to 16 out of $41(39 \%)$ cases nationwide. Comparisons between fellow eye detachment rates are limited by the small numbers observed and the varying duration of follow up. However, allowing for these limitations, the incidence of fellow eye detachments at our institution is low without prophylactic treatment. A recent Cochrane review concluded that there was insufficient evidence and in particular an absence of prospective randomized controlled trials to support or refute fellow eye prophylaxis. ${ }^{29}$

Silicone oil remains the most common choice of tamponade agent when managing GRTs, being used in $\sim 84 \%$ in this series. Its proponents advocate its use primarily because of the higher reported incidence of PVR, in addition to a perceived reduction of intraoperative slippage. Alternate tamponade agents including intraocular gas and perfluoro- $n$-octane $e^{30}$ have been utilized with reported good visual outcomes. ${ }^{10}$

Two large prospective studies published PVR incidences of $38.2 \%{ }^{9}$ and $40.7 \%{ }^{6}$

In our series, we observed a $10.0 \%$ incidence of established PVR (Grade $\mathrm{C}^{27}$ ) at presentation and this rate is similar to that recently reported in the UK epidemiology study (11.3\%). ${ }^{4}$ The overall observed rate of established PVR over the period studied was $16.9 \%$, with a proportion of patients subsequently developing 'new' PVR at RD recurrence. Our data, along with that most recently reported, suggests a lower rate of proliferative vitreoretinopathy associated with GRTs. This finding questions the necessity to use silicone oil when the strength of its use has been predicated on high associated PVR rates.

Our study supports this move away from silicone oil, particularly in eyes with fovea-sparing detachments. Patients managed with gas tamponade achieved a better visual outcome with fewer complications. There was no significant difference in anatomical success rate, nor was there a difference in the number of procedures to achieve success. Although there was trend towards a lower primary success rate in the gas group (66.7 vs $79.6 \%$ ) this was not statistically significant.

The observed difference in visual outcome is interesting. 11 of 15 patients (73.3\%) managed with gas achieved a final visual of acuity of $6 / 12$ compared to only
18 of $49(36.7 \%)$ in the oil group. The commonest cause of poor vision in eyes managed with oil was unexplained visual loss upon its removal and accounted for 10 of 49 eyes $(20.4 \%)$. This phenomenon, originally reported in $2004,{ }^{20}$ remains a concern and further supports a move away from using oil in fovea-sparing GRTs. A recently published study by authors in our group estimated the overall incidence of unexplained visual loss with silicone oil to be $3.3 \%$, with a markedly higher incidence observed in fovea-sparing GRTs. ${ }^{25}$ This is in keeping with the findings of this study involving larger numbers over a longer follow-up period.

We acknowledge limitations of this retrospective review. Analyses were performed to adjust for baseline variables potentially associated with visual outcome, and only the tamponade choice achieved statistical significance. However, this does not account for eyes where decisions to use oil were made purely on the basis of intraoperative findings (eg, retinal slippage), although we would expect this number to be small.

The risk of retinal slippage can be minimised using silicone oil ${ }^{31}$ or perfluorocarbon liquid ${ }^{10}$ for tamponade as an intraoperative air exchange can be avoided. This step is unavoidable when using gas and is therefore an obvious disadvantage, particularly in larger tears.

Additionally, there was a significant disproportionate number of GRTs extending inferiorly (between 4 and 8 o' clock) in the oil group compared to the gas group; 33.3 vs $90.2 \%$. This limits our ability to extrapolate our findings to include GRTs of any position in this study although interestingly it was not a statistically significant variable in the multiple variable regression analysis

In order to corroborate the observed beneficial effect of gas tamponade in fovea-sparing RDs, a prospective randomized controlled clinical trial appears justified. In the interim, we have generally moved away from the routine use of silicone oil towards expansile gas as post-operative tamponade in the management of GRT. We would recommend a multicentre approach to investigate this further.

\section{Summary}

What was known before

- Silicone Oil is the most commonly used tamponade agent for managing giant retinal tears (GRT).

What this study adds

- GRT-related fovea-sparing retinal detachments managed with gas tamponade achieved a significantly better visual outcome with fewer complications and no statistically significant difference in anatomical outcome.

\section{Conflict of interest}

The authors declare no conflict of interest. 


\section{References}

1 Schepens CL, Dobble JG, Mc MJ. Retinal detachments with giant breaks: preliminary report. Transactions-American Academy of Ophthalmology and Otolaryngology. American Academy of Ophthalmology and Otolaryngology 1962; 66: 471-479.

2 Scott JD. Giant tear of the retina. Transactions of the ophthalmological societies of the United Kingdom 1975; 95: 142-144.

3 Kanski JJ. Giant retinal tears. Am J Ophtalomol 1975; 79: 846-852.

4 Ang GS, Townend J, Lois N. Epidemiology of giant retinal tears in the United Kingdom: the British Giant Retinal Tear Epidemiology Eye Study (BGEES). Investigative ophthalmology \& visual science 2010; 51: 4781-4787.

5 Chang S, Lincoff H, Zimmerman NJ, Fuchs W. Giant retinal tears. Surgical techniques and results using perfluorocarbon liquids. Arch Ophthalmol 1989; 107: 761-766.

6 Kertes PJ, Wafapoor H, Peyman GA, Calixto Jr N, Thompson $\mathrm{H}$. The management of giant retinal tears using perfluoroperhydrophenanthrene. A multicenter case series. Vitreon Collaborative Study Group. Ophthalmology 1997; 104: 1159-1165.

7 Kreiger AE, Lewis $\mathrm{H}$. Management of giant retinal tears without scleral buckling. Use of radical dissection of the vitreous base and perfluoro-octane and intraocular tamponade. Ophthalmology 1992; 99: 491-497.

8 Verstraeten T, Williams GA, Chang S, Cox Jr MS, Trese MT, Moussa $\mathrm{M}$ et al. Lens-sparing vitrectomy with perfluorocarbon liquid for the primary treatment of giant retinal tears. Ophthalmology 1995; 102: 17-20.

9 Scott IU, Murray TG, Flynn Jr HW, Feuer WJ, Schiffman JCPerfluoron Study Group. Outcomes and complications associated with giant retinal tear management using perfluoro-n-octane. Ophthalmology 2002; 109: 1828-1833.

10 Sirimaharaj M, Balachandran C, Chan WC, Hunyor AP, Chang AA, Gregory-Roberts J et al. Vitrectomy with short term postoperative tamponade using perfluorocarbon liquid for giant retinal tears. Br J Ophthalmol 2005; 89: 1176-1179.

11 Lee SY, Ong SG, Wong DW, Ang CL. Giant retinal tear management: an Asian experience. Eye 2009; 23: 601-605.

12 Karel I, Michalickova M, Soucek P. Long-term results of pars plana vitrectomy and silicone oil for giant tears. Eur J Ophthalmol 1996; 6: 315-321.

13 Ghosh YK, Banerjee S, Savant V, Kotamarthi V, Benson MT, Scott RA et al. Surgical treatment and outcome of patients with giant retinal tears. Eye 2004; 18: 996-1000.

14 Unlu N, Kocaoğlan H, Acar MA, Sargin M, Aslan BS, Duman $\mathrm{S}$. The management of giant retinal tears with silicone oil. Eur J Ophthalmol 2003; 13: 192-195.

15 Aylward GW, Cooling RJ, Leaver PK. Trauma-induced retinal detachment associated with giant retinal tears. Retina 1993; 13: 136-141.
16 Leaver PK, Billington BM. Vitrectomy and fluid/silicone-oil exchange for giant retinal tears: 5 years follow-up. Graefes Arch Clin Exp Ophthalmol 1989; 227: 323-327.

17 Goezinne F, LA Heij EC, Berendschot TT, Gast ST, Liem AT, Lundqvist IL et al. Low redetachment rate due to encircling scleral buckle in giant retinal tears treated with vitrectomy and silicone oil. Retina 2008; 28: 485-492.

18 Ambresin A, Wolfensberger TJ, Bovey EH. Management of giant retinal tears with vitrectomy, internal tamponade, and peripheral 360 degrees retinal photocoagulation. Retina 2003; 23: 622-628.

19 Kapetanios AD, Donati G, Pournaras CJ. [Idiopathic giant retinal tears: treatment with vitrectomy and temporary silicone oil tamponade]. J Francias Ophtalomol 2000; 23: 1001-1005.

20 Newsom RS, Johnston R, Sullivan PM, Aylward GB, Holder GE, Gregor ZJ. Sudden visual loss after removal of silicone oil. Retina 2004; 24: 871-877.

21 Newsom RS, Johnston R, Sullivan P, Aylward B, Holder G, Gregor Z. Visual loss following silicone oil removal. $\mathrm{Br} J$ Ophthalmol 2005; 89: 1668.

22 Cazabon S, Groenewald C, Pearce IA, Wong D. Visual loss following removal of intraocular silicone oil. $\mathrm{Br} J$ Ophthalmol 2005; 89: 799-802.

23 Herbert EN, Laidlaw DA, Williamson TH, Habib M, Steel D. Loss of vision once silicone oil has been removed. Retina 2005; 25: 808-809, author reply-809.

24 Williams PD, Fuller CG, Scott IU, Fuller DG, Flynn HW. Vision loss associated with the use and removal of intraocular silicone oil. Clin Ophtalomol 2008; 2: 955-959.

25 Moya R, Chandra A, Banerjee PJ, Tsouris D, Ahmad N, Charteris DG. The incidence of unexplained visual loss following removal of silicone oil. Eye 2015; 29: 1477-1482.

26 Batman C, Cekic O. Vitrectomy with silicone oil or longacting gas in eyes with giant retinal tears: long-term followup of a randomized clinical trial. Retina 1999; 19: 188-192.

27 Machemer R, Aaberg TM, Freeman HM, Irvine AR, Lean JS, Michels RM. An updated classification of retinal detachment with proliferative vitreoretinopathy. Am J Ophtalomol 1991; 112: 159-165.

28 Wickham L, Connor M, Aylward GW. Vitrectomy and gas for inferior break retinal detachments: are the results comparable to vitrectomy, gas, and scleral buckle? $\mathrm{Br} \mathrm{J}$ Ophthalmol 2004; 88: 1376-1379.

29 Ang GS, Townend J, Lois N. Interventions for prevention of giant retinal tear in the fellow eye. The Cochrane database of systematic reviews 2012; 2: CD006909.

30 Bottoni F, Bailo G, Arpa P, Prussiani A, Monticelli M, de Molfetta V. Management of giant retinal tears using perfluorodecalin as a postoperative short-term vitreoretinal tamponade: a long-term follow-up study. Ophthalm Surg 1994; 25: 365-373.

31 Dabour SA. The outcome of surgical management for giant retinal tear more than 180 degrees. BMC Ophthalmol 2014; 14: 86 . 